\title{
Free Rotor Styrylcyanine Chromogens
}

\author{
M. M. HABASHY, M. S. ANTONIOUS, M. ABDEL-KADER \\ and M. S. A. ABDEL-MOTTALEB $\dagger$
}

Department of Chemistry, Faculty of Science, Ain Shams University, Abbassia, Cairo, A.R. Egypt

(Received October 22, 1985)

Fluorescence spectra (maximum wavelength $\lambda_{F}$ ) and fluorescence quantum yields $\left(\phi_{F}\right)$ were measured for four structurally related styrylcyanine chromogens of the pyridinium and quinolinium type (1-4) in different solvents at ambient temperature and $77 \mathrm{~K}$. The response of $\lambda_{F}$ and $\phi_{F}$ values to changes in solvent polarity, solvent hydrogen bonding donor strength, viscosity and temperature was a sensitive function of chromogen structure. The sensitivities of the $\lambda_{F}$ and $\phi_{F}$ values correlate with the degree of charge transfer character of the $S_{1, C T}$ state; Stokes shift of fluorescence was progressively decreased while $\phi_{F}$ value was enhanced as the $C T$ character of $S_{1, C T}$ state increases. Moreover, a large edge-excitation red shift was observed in ethanol glass at $77 \mathrm{~K}$. The dominant photophysical features for these dyes are discussed in terms of strong emission from an intramolecular CT state characterized by different solvation sites indicated by the observation of the excitation-wavelength dependent phenomenon in ethanol at $77 \mathrm{~K}$ and an important non-radiative decay channel involving rotation of the different parts of molecules leading to a more relaxed weakly fluorescent $S_{1, C T}$ created in fluid media. The viscosity dependence of fluorescence properties (a marked increase in $\phi_{F}$ was observed with increasing viscosity) suggests that these dyes can be useful reporters of microviscosity for different sites in various organized assemblies. Moreover, it was suggested that increasing $\mathrm{H}$-bonding donor strength of the solvent activates a rotatory non-radiative decay channel probably by localizing charge densities and decreasing $C T$ nature of the $S_{1, C T}$ state.

KEY WORDS: Fluorescence spectra and quantum yields; Styrylcyanine chromogens; Charge transfer.

\section{INTRODUCTION}

Recent years have witnessed an increasing interest in the photophysical relationship between fluorescence properties of free rotor chromogens of important modern applications and the molecular structure, solvent viscosity and temperature. ${ }^{1-5}$

$\dagger$ Author to whom all correspondence should be addressed. 
It has been previously shown that the energy and yield of fluorescence emission for a negatively solvatochromic styrylcyanine chromogen (1) of the electron donor-acceptor type, representing an important class of fluorescence probe dyes, ${ }^{1,6,7}$ depend critically on solvent viscosity. ${ }^{1,7}$ A model of nonradiative decay has been proposed. ${ }^{1}$ This model employs a planar highly emissive intramolecular $C T$ excited state $\left(S_{1, C T}\right)$ capable of decay to a weakly fluorescent twisted $C T$ state by radiationless isomerization process involving rotation of the different parts of the molecule; a hindered rotor effect. Intersystem crossing to the triplet states was considered inefficient. The viscosity dependence of photophysical properties suggested that this molecule can be a useful sensor of microviscosity for different sites in various organized assemblies. ${ }^{1,7}$

In continuation of our work we report in the present paper further studies on the fluorescence properties of four structurally related styrylcyanine chromogens (1-4) in three solvents; ethanol, $\mathrm{CH}_{2} \mathrm{Cl}_{2}$, and glycerol representing three different classes of solvents, namely hydrogen bonding donor, non-hydrogen bonding solvent and highly viscous solvent, respectively. The measurements are performed at
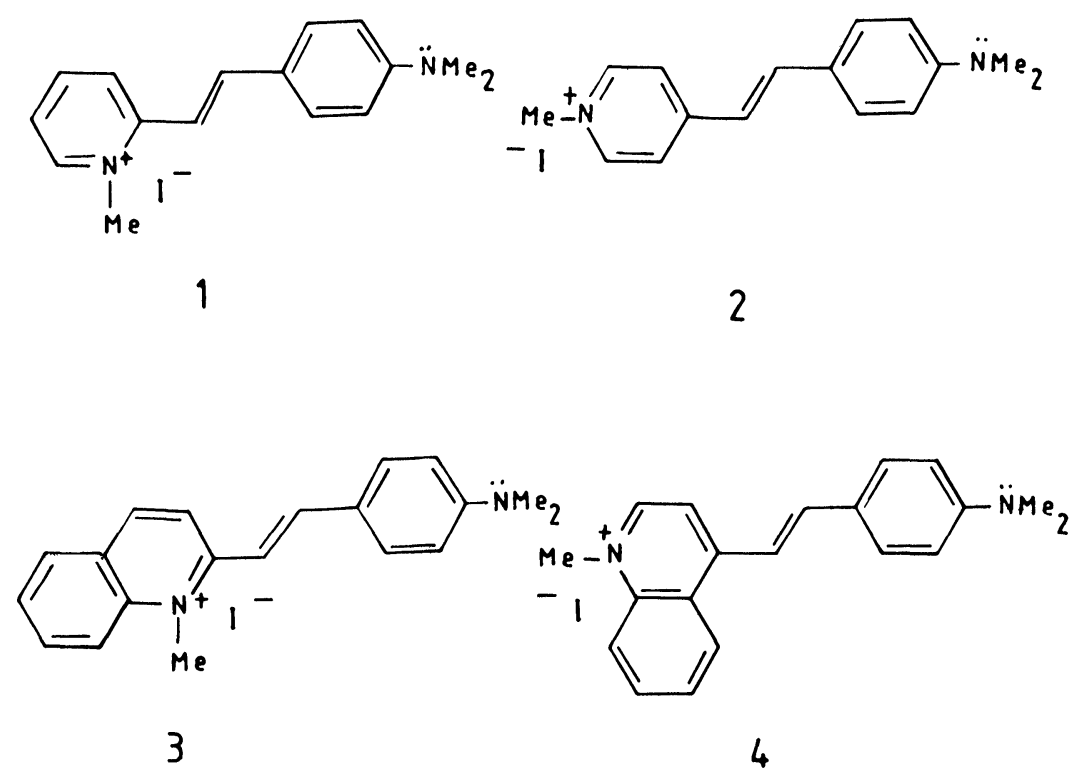
ambient temperature and $77 \mathrm{~K}$. The results have a significant bearing on the structure requirements for engineering deactivation pathways of the $S_{1, C T}$. Moreover, the role of solvation-site heterogeneity ${ }^{8}$ in the fluorescence spectra of compounds $1-4$ in ethanol at $77 \mathrm{~K}$, were examined.

\section{EXPERIMENTAL}

Styrylcyanine chromogens (1-4) were prepared by condensing the pyridinium and quinolinium salts, namely 1,2-dimethylpyridinium-, 1,4-dimethylpyridinium, 1,2-dimethylquinolinium- and 1,4-dimethylquinolinium iodides, respectively, with an excess amount of $p-N, N$ dimethylamino-benzaldehyde in the presence of piperidine as a basic catalyst. Their purities were checked by $T L C$ on silica gel with methanol as an eluent.

Spectroscopic pure solvents were used for all measurements. Absorption spectral data were obtained on a SP-1800 Pye Unicam spectrophotometer. Fluorescence spectra were taken on a Shimadzu 510 spectrofluorimeter. Fluorescence quantum yields were determined by comparing with a fluorescein fluorescence standard $\left(\phi_{F}=0.85\right)$. Preliminary measurements showed that degassing of the chromogen solution has practically no effect on the $\phi_{F}$ value, a result which eliminates the possibility of bimolecular quenching by dissolved oxygen.

\section{RESULTS AND DISCUSSION}

Earlier work on styrylcyanine and merocyanine-like dye-stuffs suggested that the longest wavelength electronic transition in the visible spectra of these negative solvatochromic compounds is due to intramolecular $C T\left(S_{0} \rightarrow S_{1}\right)$ transition. ${ }^{6,8,9}$ The spectra of the compounds under investigation exhibit a similar intramolecular $C T$ transition characterized by a decreasing $C T$ energy, E (CT), in the sequence $1>2>3>4$. This parameter has been used as a convenient gauge of the degree of $C T$ in the first singlet excited state of $D-A$ class compounds. ${ }^{10}$ This trend is further supported by diminution of halfband width, $\Delta \bar{\nu}_{1 / 2}$, and enhancement of molar absorptivity, $\varepsilon_{\max }$, in the same sequence mentioned above. These observations indicate that 
the electronic distribution within these molecules tends to symmetrize ${ }^{6}$ (become less polar) in the order $1>2>3>4$ in their $S_{0}$ states so that optical excitation to the $S_{1, C T}$ state occurs with decreasing changes in their electronic distribution in the sequence $1>2>3>4$. In other words, optical pumping occurs with a decreasing degree of $C T$ as the sequence above. The wavelength and quantum yield of fluorescence of chromogens (1-4) are sensitive to changes in solvent viscosity, temperature and excitation wavelength. Therefore, fluorescence spectra and quantum yield determinations were made in different media at room temperature and at $77 \mathrm{~K}$ (Table I).

At ambient temperature, changing the solvent from non-viscous ethanol to glycerol has the expected effect on the fluorescence properties. For a given dye molecule, the Stokes shift of fluorescence decreases, reflecting a decrease in geometry difference between ground state and the emitting state. Moreover, $\phi_{F}$ is enhanced markedly due to an increasing lifetime of the $S_{1, C T}$ state by increasing the contribution of the solvent-dependent viscosity barrier to a weakly fluorescent state. $^{1}$

Interestingly, about three orders of magnitude increase in $\phi_{F}$ for dyes 1-4 in ethanol on going from ambient temperature (fluid medium) to $77 \mathrm{~K}$ (rigid medium) was observed. Furthermore, a pronounced decrease in Stokes shift was observed as the temperature is lowered to $77 \mathrm{~K}$. Moreover, once the solution is made rigid, excitationwavelength dependence was observed for dyes 1-4. The fluorescence spectra of a dilute solution $\left(\sim 10^{-6} \mathrm{M}\right)$ of dye (4) in ethanol at $77 \mathrm{~K}$ as a function of excitation wavelength are shown in Figure 1. A large red shift in the range $(800-1000) \mathrm{cm}^{-1}$ for dyes $1-4$ has been observed as the excitation wavelength is tuned from the absorption maximum to about $40 \mathrm{~nm}$ higher to the red edge of the absorption band. However, the fluorescence spectra of dilute solutions of dyes 1-4 at room temperature are excitation wavelength independent.

In order to explain these observations we refer to a qualitative excited state potential energy surface (Figure 1b) representing the solvent interaction with the flexible dye molecules that may assume different rotational conformation. ${ }^{1,8}$ It is reasonable to assume a statistical distribution of the solute among different solvation sites. ${ }^{8}$ In rigid ethanol at $77 \mathrm{~K}$ the lifetime of the various solvation sites is large compared to the excited state lifetime due to the existence of high solvent-dependent viscosity and torsional barriers, so that dissipation 


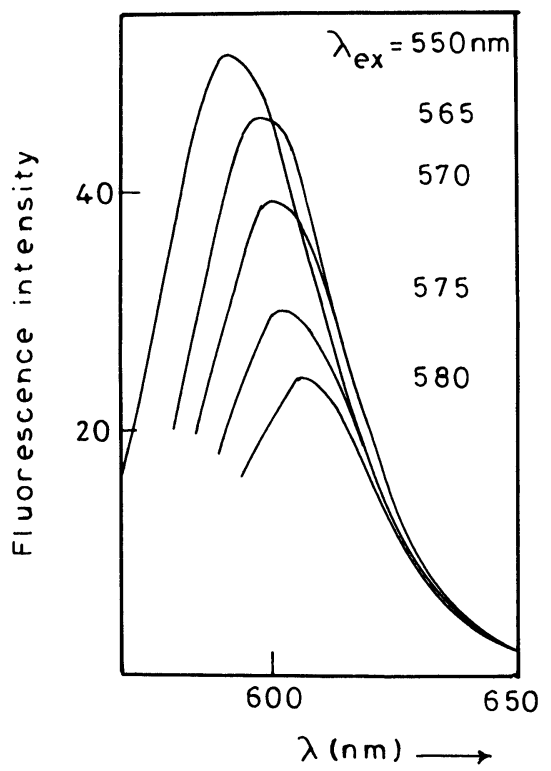

FIGURE 1a Excitation-wavelength-dependent fluorescence spectra of chromogen (4) in ethanol glass at $77 \mathrm{~K}$ (Red edge effect). Tuning $\lambda_{\text {exc }}$ from the absorption maximum $(550 \mathrm{~nm})$ to the red edge of the absorption band results in a red shift of $\lambda_{F}$.

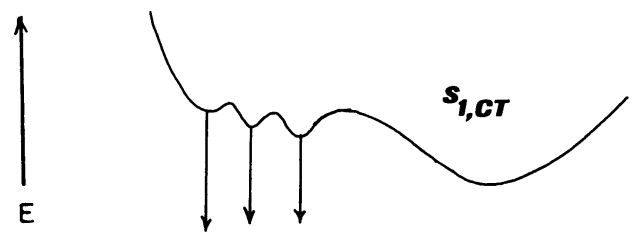

FIGURE 1b Postulated potential energy surface for different solvation sites in rigid medium.

of electronic energy by crossing these barriers is considerably prohibited. Excitation of the dye molecules in any specific site gives rise to a sudden strained Franck-Condon state leading to a fast solutesolvent relaxation to the equilibrium excited state of this specific site, from which strong emission originates. This accounts for the observed high $\phi_{F}$ and small Stokes shift ${ }^{1}$ in the rigid medium. 
M. M. HABASHY et al.

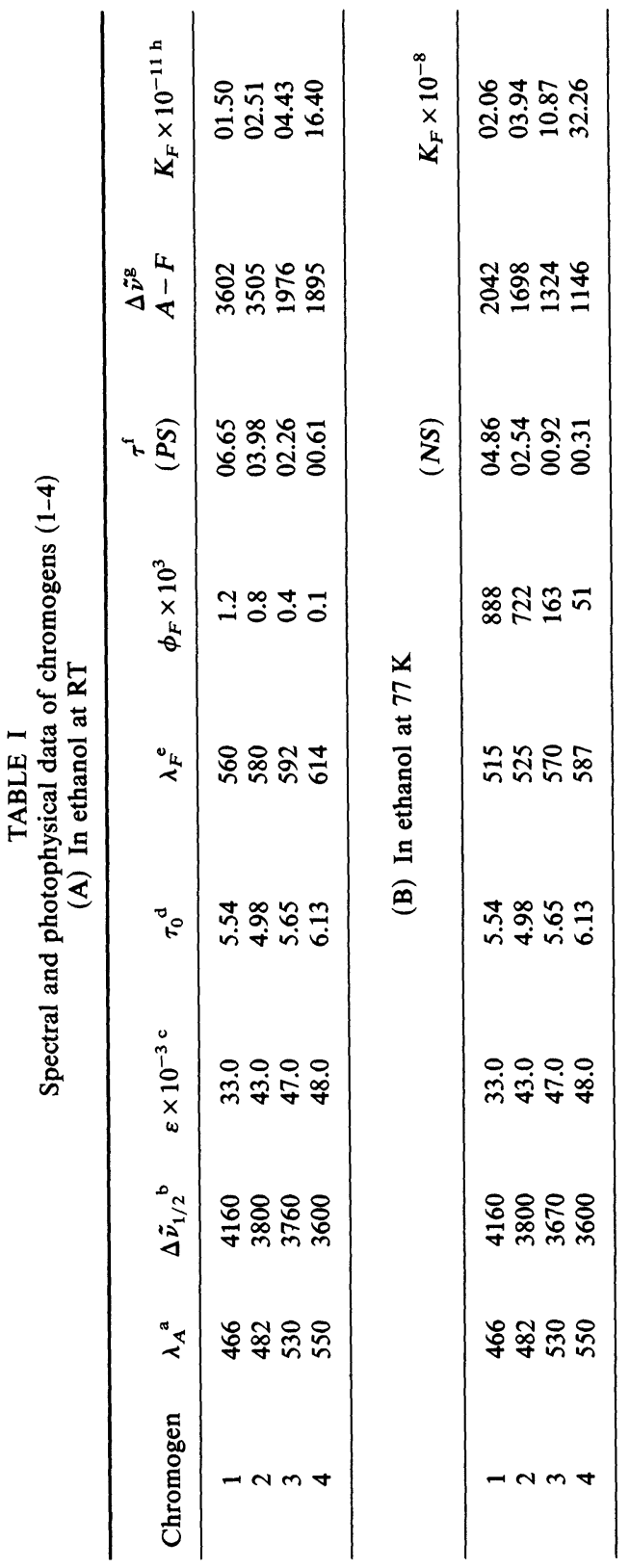


FREE ROTOR STYRYLCYANINE CHROMOGENS

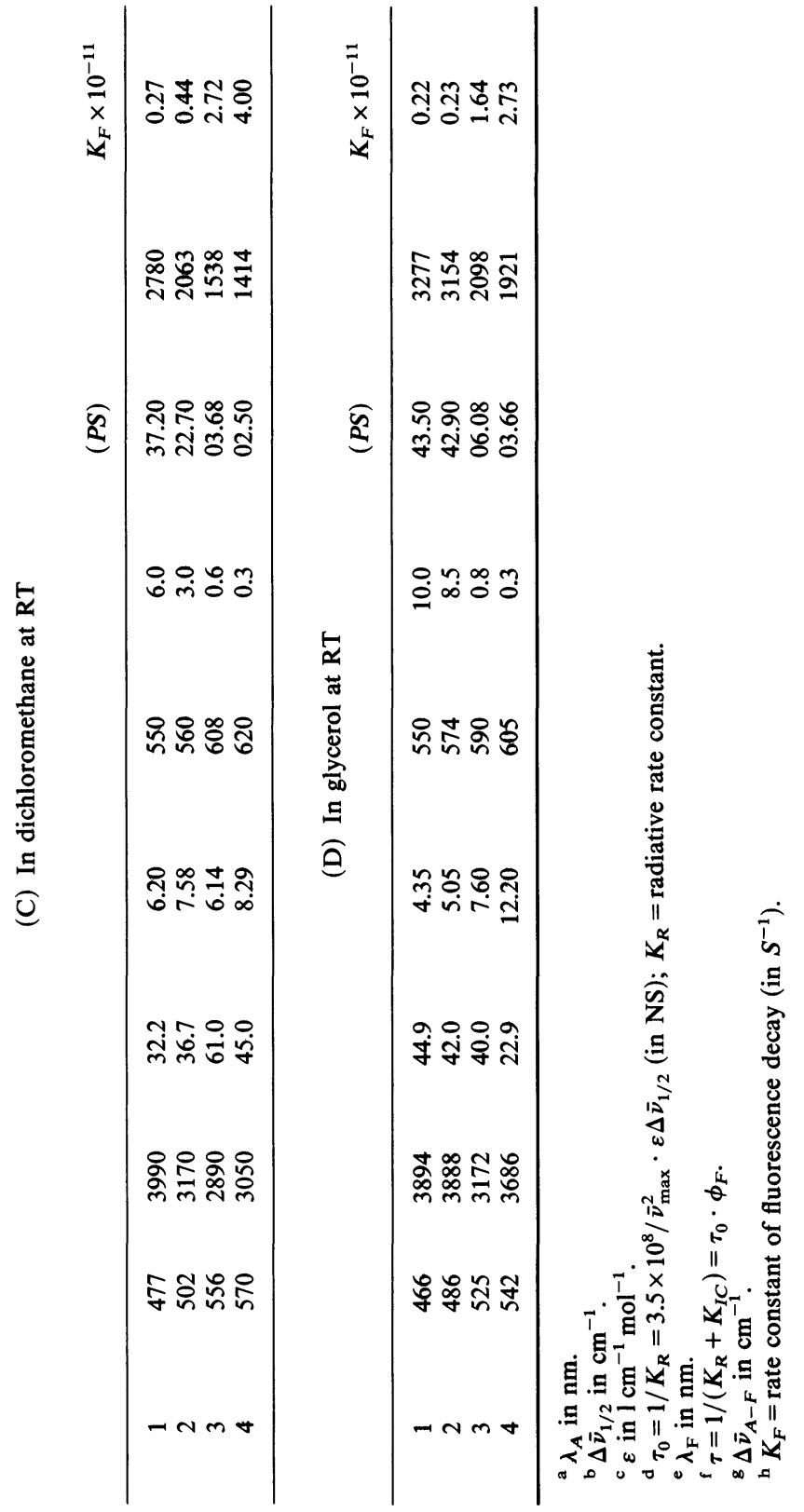


In fluid ethanol the lifetime of the various solvation sites is short compared to the excited state lifetime and a dynamic equilibrium exists, leading to a weak emission from the most stable solvation site indicated by larger Stokes shift. Emission will occur at the same wavelength independent of the excitation wavelength.

The principal structure feature affecting photophysical properties noted here is the role of the electron-acceptor cationic heterocyclic groups in 1-4 which results in a systematic differential response to changes in solvent properties and temperature (Table I). The change in emission spectral properties of dyes 1-4 could be readily understood in terms of the degree of intramolecular $C T$ which involves promotion of electronic charge from an orbital which is significantly weighted at the donor part of the molecule, particularly at the $\mathrm{N}, \mathrm{N}$-dimethylamino group. ${ }^{6}$

The greater $C T$ character of the $S_{1, C T}$ state of (1) and (2) relative to (3) and (4) is associated with substantial alteration of the electron densities and bond orders ${ }^{6}$ which results in pronounced different response to medium effects. As indicated by the spectral observations above mentioned, it could be concluded that, a larger contribution from the locally excited state of molecules (3) and (4) lowers the barrier to rotation which ensures that the excited state molecules travel faster to the most favourable geometry for nonradiative decay to the $S_{0}$ state.

Finally changing the solvent from a non-hydrogen bonding $\mathrm{CH}_{2} \mathrm{Cl}_{2}$ to ethanol $(\alpha \text {-value }=0.850)^{11}$ also activates extensive rotatory decay probably due to increasing specific $H$-bonding interaction with the $S_{1, C T}$ state in the sequence $1<2<3<4$. This accounts for the larger Stokes shift $\left(\Delta \bar{\mu}_{A-F}\right)$ and smaller $\phi_{F}$ value in ethanol (Table I).

Similar to a previously found correlation ${ }^{5}$ it has been found that, in a given medium the size of the Stokes shift of fluorescence $\left(\Delta \bar{\nu}_{A-F}\right)$ is a measure of $C T$ nature of the $S_{1, C T}$ state of dyes. The greater the $C T$ nature of the $S_{1, C T}$ state, the smaller is the probability that rotational-viscosity barrier can be crossed.

The rate constants of internal conversion determined from the difference in decay rates in ethanol at room temperature and at $77 \mathrm{~K}$ are so close to $K_{f}$ in value and increases as the degree of $C T$ character decreases in the order 1-4 (Table 1, Section A). The results suggest that these molecules can be used as fluorescent indicators to study microscopic structural detail in organized assemblies., ${ }^{72}$ 


\section{References}

1. M. S. A. Abdel-Mottaleb, Laser Chem. 4, 305 (1984) and references therein.

2. (a) Z. R. Grabowski, K. Rotkiewicz, A. Siemiarczuk, D. Cowley and W. Baumann. Nov. J. Chim. 3, 443 (1979). (b) Z. R. Grabowski and J. Dobkowski, J. Pure Appl. Chem. 55, 245 (1983).

3. H. E. Zimmerman and M. G. Steinmetz, J. Chem. Soc., Chem. Commun. 230 (1978) and references therein.

4. M. J. Snare, F. E. Treloar, K. P. Ghiggino and P. J. Thistlethwaite, J. Photochem. 18, 335 (1982).

5. G. Jones, W. R. Jackson and C. Y. Choi, J. Phys. Chem. 89, 294 (1985).

6. M. S. A. Abdel-Mottaleb, Z. Phys. Chem. (Leipzig) 264, 957 (1983).

7. J. K. Thomas, Chem. Rev. 80, 283 (1980).

8. K. A. Al-Hassan and M. A. El-Bayoumi, Chem. Phys. Lett. 76, 121 (1980).

9. M. S. A. Abdel-Mottaleb, Z. Naturforsch. 37A, 1353 (1982).

10. T. P. Carsey, G. L. Findley and S. P. McGlynn, J. Am. Chem. Soc. 101, 4502 (1979).

11. R. W. Taft and H. J. Kamlet, J. Am. Chem. Soc. 98, 2886 (1976).

12. N. J. Turro, M. Gratzel and A. M. Braun, Angew. Chem. Int. Ed. Engl. 19, 675 (1980). 